Proc. XII Int. School on Theoretical Physics — Symmetry and Structural Properties of Condensed Matter

\title{
Wetting of Nanostructurized Sapphire and Gold Surfaces
}

\author{
M. Kempiński ${ }^{a, b}$, J. JenCzYK ${ }^{b}$, S. JURGA ${ }^{a, b}$ And M. ŚliwińskA-BARTKOWiAK ${ }^{a}$ \\ ${ }^{a}$ Faculty of Physics, Adam Mickiewicz University, Umultowska 85, 61-614 Poznań, Poland \\ ${ }^{b}$ NanoBioMedical Centre, Adam Mickiewicz University, Umultowska 85, 61-614 Poznań, Poland
}

We present the results of preliminary experiments regarding research on the contact angle measurements of various liquids on solid surfaces with different morphology. The aim was to get insight into the dependence of wetting phenomena on the nanoscale surface roughness. Flat and nanostructurized surfaces of gold and sapphire were used in the experiments. Four liquids - bromobenzene, water, mercury, and gallium — covering a broad range of surface tension values were used to check how varying roughness influences wetting in the systems with different adhesion/cohesion ratio. Structurization was anisotropic, which resulted in the very interesting behaviour of the examined liquids on the selected surfaces. Significant change of the wetting properties was observed as well as a strong dependence on the surface morphology.

DOI: 10.12693/APhysPolA.132.185

PACS/topics: 68.08.Bc, 68.35.Np, 68.35.Ct

\section{Introduction}

Wetting of solid surfaces with liquids is an important phenomenon in life, science and engineering areas, such as: capillary effects, coatings, microfluidics, surface physics, and chemistry.

For the last several years most of the work concerning wetting has been devoted to the behavior of water. As water is the most common liquid of everyday life, thus gaining control on the hydrophobicity (or hydrophilicity) of the surface is crucial for many applications [1, 2].

Especially interesting area concerns patterned surfaces, where wetting strongly depends on the surface roughness and can be directional $[3,4]$.

The behavior of various liquids in contact with rough solid surfaces is also very important from the point of view of the adsorption in porous materials, because pore walls cannot be considered "flat". It was already shown that the pore size and geometry have significant influence on the phenomena occurring inside pores such as phase transitions and chemical reactions [5-7] and that contact angle can be correlated with the microscopic wetting parameter $\alpha[8]$.

The definition of wetting states as "the ability of a liquid to maintain contact with a solid surface, resulting from the intermolecular interactions" is most widely used. The degree of wetting is determined by the balance between the adhesive and cohesive forces.

In the most simple approach the thermodynamic equilibrium between the three phases $(S$ - solid, $L$ - liquid and $G$ - gas) can be described with the Young equation

$$
0=\gamma_{S G}-\gamma_{S L}-\gamma_{L G} \cos \theta_{C},
$$

where $\gamma$ is the surface energy (surface tension) and $\theta_{C}$ is the contact angle.

Knowing the contact angle and using the Young-Dupre equation: $W_{a}=\gamma_{L G}\left(1+\cos \theta_{C}\right)$ we can calculate the work of adhesion, which gives us the energy of adhesion between the liquid droplet and the solid surface, i.e. the work required to separate the liquid droplet from the surface, leaving the adlayer on the solid surface [9].
In our research we focus on the influence of the nanoscale roughness on the wetting of the surface with liquids. In order to obtain a general view on the phenomenon we use surfaces made of different materials as well as different liquids, which should provide us with information on the impact of roughness on the so-called wetting and non-wetting systems.

This work concerns sapphire and gold surfaces on top of which droplets of liquid bromobenzene $\left(\mathrm{C}_{6} \mathrm{H}_{5} \mathrm{Br}\right)$, water $\left(\mathrm{H}_{2} \mathrm{O}\right)$, mercury $(\mathrm{Hg})$, and gallium $(\mathrm{Ga})$ were placed. The motivation behind this choice is that:

- sapphire crystal cut along a specific crystallographic directions forms unstable surfaces, which can be structurized on the nanoscale level in the easy and controlled way, thus allowing us to follow the changes of wetting due to the surface roughness. Additionally this structurization is strictly directional (anisotropic), leading to the very interesting behaviour of the adsorbed liquids. Moreover, sapphire seems a very promising material in the future engineering, due to its high hardness and transparency as well as chemical and thermal resistance;

- gold is relatively easy to deposit on any other solid surface (sapphire in our case) with the vacuum thermal deposition technique and it is chemically inert, thus does not change (oxidize) and retains the surface morphology after removing the sample from the vacuum deposition chamber

- liquids selected for this work cover a broad range of surface tension values in air and this allows for comparison between the systems with different adhesion/cohesion ratio.

\section{Experimental}

First stage of the experiment involved the preparation of four types of surfaces: flat sapphire, nanostructurized sapphire, flat gold, and nanostructurized gold.

One side epi-polished $\alpha$-sapphire cut along the $M$ plane was obtained from CrysTec $\mathrm{GmbH}$. Cutting along the $M$-plane produces an unstable surface of singular $\alpha-\mathrm{Al}_{2} \mathrm{O}_{3}(10-10)$. Annealing in temperatures above 
$1300^{\circ} \mathrm{C}$ leads to the formation of the faceted, V-shaped nanogrooves [10]. To obtain a gold surface of the similar topography, $100 \mathrm{~nm}$ thick gold layer was deposited on both flat and nanostructurized $\mathrm{Al}_{2} \mathrm{O}_{3}$ using the PREVAC ultra-high vacuum electron beam evaporation system.

The second stage comprised the contact angle measurements performed using the SEO Contact Angle Analyzer Phoenix 300 in ambient conditions (air, room temperature, atmospheric pressure). Four liquids were examined, with the following surface tension values (versus air): $\mathrm{C}_{6} \mathrm{H}_{5} \mathrm{Br}\left(\gamma_{L G}=36.5 \times 10^{-3} \mathrm{~N} / \mathrm{m}\right)[11], \mathrm{H}_{2} \mathrm{O}\left(\gamma_{L G}=\right.$ $\left.72.8 \times 10^{-3} \mathrm{~N} / \mathrm{m}\right)[11], \mathrm{Hg}\left(\gamma_{L G}=435.5 \times 10^{-3} \mathrm{~N} / \mathrm{m}\right)[12]$ and $\mathrm{Ga}\left(\gamma_{L G}=724 \times 10^{-3} \mathrm{~N} / \mathrm{m}\right)$ [13]. Contact angle was measured on four different surfaces with double measurement on each nanostructurized sample (parallel and perpendicularly to the linear features). Contact angle was determined with the accuracy of approximately $0.5^{\circ}$. Every measurement was repeated several times at different locations of surface. Droplets were allowed to stabilize on the surface before contact angle was measured, to ensure equilibrium conditions.

Because the $\mathrm{Hg}$ droplets completely dissolved the gold layer directly underneath in the process of amalgamation we tried to use Ga which, similarly to mercury, poorly wets solid surfaces. In this case the diffusion of Ga atoms inside the Au layer was observed (alloying), again affecting the wetting phenomena. Thus the results for $\mathrm{Hg}$ and $\mathrm{Ga}$ on $\mathrm{Au}$ could not be unambiguously interpreted and are not present in this article.

\section{Results and discussion}

The wetting behavior can be very well observed in the measurements of the contact angle of a liquid droplet placed on a solid surface. Generally, liquids with low surface tension show better wetting of the solid surface than the high-surface tension ones. Figure 1 shows examples of good wetting (low contact angle) and poor wetting (high contact angle) systems. The other factor im-
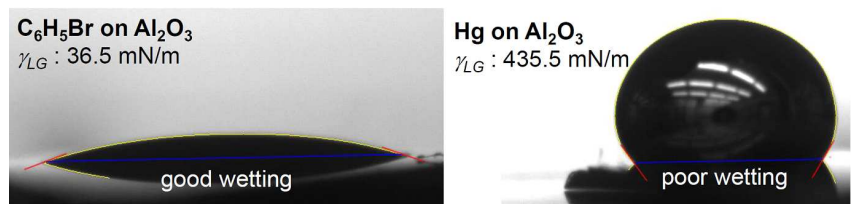

Fig. 1. $\mathrm{C}_{6} \mathrm{H}_{5} \mathrm{Br}$ (left) and $\mathrm{Hg}$ (right) on the same surface (flat sapphire) show different behaviour which depends on the adhesion/cohesion ratio. Contact angle $\theta_{C}$ is approximately $20^{\circ}$ for $\mathrm{C}_{6} \mathrm{H}_{5} \mathrm{Br}$, while for mercury it rises to $135^{\circ}$.

portant for the wetting phenomena is the adhesion of a liquid to the solid surface. Adhesion depends mostly on the intermolecular forces between a liquid and a solid, so it directly relies on the materials used, but may also change with the shape of the solid-liquid interface. In most cases this will be determined by the solid surface topography. For this reason we selected four different surfaces for this work. Figure 2 shows the atomic force microscopy (AFM) images of the samples together with the values of a root mean square (rms) parameter which describes the surface roughness.
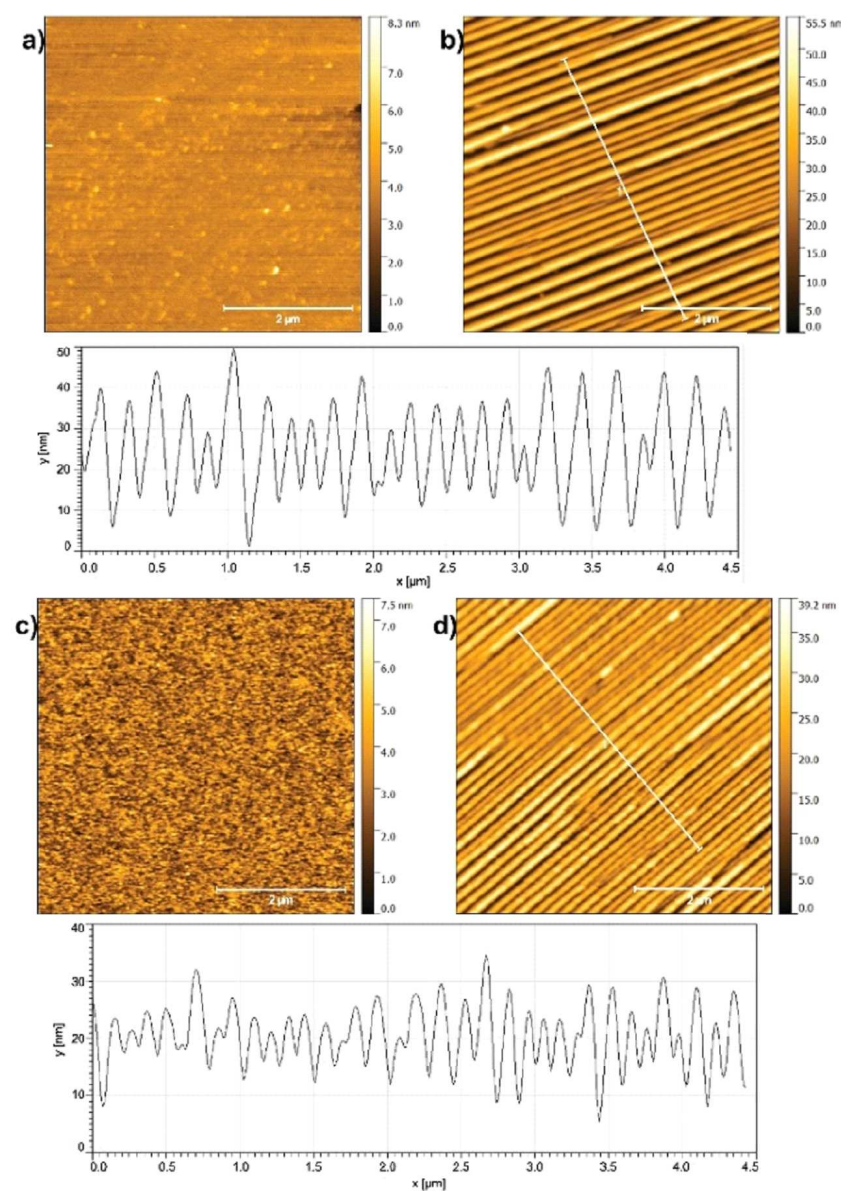

Fig. 2. AFM topography images of: (a) flat (as received) $\alpha-\mathrm{Al}_{2} \mathrm{O}_{3}, \mathrm{rms}=1.10 \mathrm{~nm}$, (b) $\alpha-\mathrm{Al}_{2} \mathrm{O}_{3}$ annealed in $T=1500^{\circ} \mathrm{C}$, rms $=11.10 \mathrm{~nm}$, with the height profile along the marked line, (c) $100 \mathrm{~nm} \mathrm{Au}$ on flat $\alpha-\mathrm{Al}_{2} \mathrm{O}_{3}$, rms $=0.97 \mathrm{~nm}$, (d) $100 \mathrm{~nm} \mathrm{Au}$ on nanostructurized $\alpha$ $\mathrm{Al}_{2} \mathrm{O}_{3}, \mathrm{rms}=6.61 \mathrm{~nm}$, with the height profile along the marked line. All images are $5 \times 5 \mu \mathrm{m}$ squares.

The grooves created on the annealed $\mathrm{Al}_{2} \mathrm{O}_{3}$ are still present after Au deposition and have similar period. We can also observe a surface smoothing after the gold deposition, probably because the Au layer is quite thick when compared to the height of the nanostructures on sapphire.

Contact angle measurements revealed clear differences in the wetting of the above surfaces. The most striking fact from this experiment is that wetting of the nanostructurized sapphire is anisotropic, which results in the elongation of a liquid droplet on the surface when viewed from above. Much different behavior was observed in the case of gold, where the direction of grooves on the surface seem to had no effect on wetting, at least for $\mathrm{H}_{2} \mathrm{O}$ and $\mathrm{C}_{6} \mathrm{H}_{5} \mathrm{Br}$. This phenomenon is depicted in Fig. 3, 
which shows water droplets placed on the nanostructurized sapphire and gold surfaces. The full set of contact angle values and work of adhesion is presented in Fig. 4.

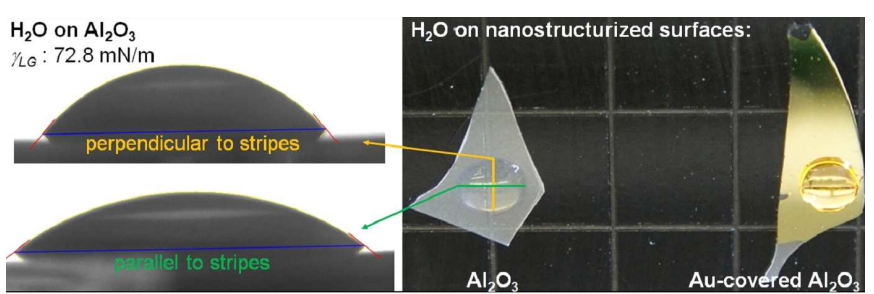

Fig. 3. $\mathrm{H}_{2} \mathrm{O}$ droplets on nanostructurized sapphire and gold. Left picture shows droplet cross-sections parallel and perpendicular to the nanogrooves, while right picture shows $\mathrm{H}_{2} \mathrm{O}$ droplets on sapphire and gold viewed from above. Droplet on sapphire is elongated, while the one on gold is round. Similar behavior was observed in case of $\mathrm{C}_{6} \mathrm{H}_{5} \mathrm{Br}$, but not as clearly visible as for water, due to the lower difference between contact angle values measured parallel and perpendicularly.

On the basis of the above data we can observe several interesting features

- $\mathrm{C}_{6} \mathrm{H}_{5} \mathrm{Br}$ shows the highest degree of wetting of all the examined surfaces, and better adhesion to gold than sapphire. $\mathrm{H}_{2} \mathrm{O}$ shows medium wetting and better adhesion to sapphire than gold. $\mathrm{Hg}$ and $\mathrm{Ga}$ are the worst wetting liquids of the used set. This observation can be easily correlated with the surface tension of the four liquids: 36.5, $72.8,435.5$, and $723.9 \mathrm{mN} / \mathrm{m}$ for $\mathrm{C}_{6} \mathrm{H}_{5} \mathrm{Br}, \mathrm{H}_{2} \mathrm{O}, \mathrm{Hg}$, and Ga, respectively.

- For $\mathrm{C}_{6} \mathrm{H}_{5} \mathrm{Br}$ and $\mathrm{H}_{2} \mathrm{O}$ on both sapphire and gold we observed enhanced wetting (decrease of the $\theta_{C}$ value), when compared to the flat surface, resulting from the increase of the surface roughness. This behaviour seems consistent with the Wenzel model [14], which states that roughening (structurization) amplifies the natural tendency of a surface. It means that e.g. a hydrophobic surface would become even more hydrophobic and a hydrophilic surface would become even more hydrophilic after the roughness increases. According to Wenzel, contact angle should satisfy the following relation: $\cos \theta_{C}=$ $r \cos \theta^{*}$, where $r$ is the roughness ratio (area of the real rough surface divided by the projected area), $\theta_{C}$ is the contact angle on the rough surface and $\theta^{*}$ is the contact angle on the flat surface. In our experiments roughness ratio values calculated from the Wenzel equation as $r=\cos \theta_{C} / \cos \theta^{*}$ do not match the values obtained from the AFM images, thus the correlation with the Wenzel model is only qualitative. There is a number of works which show that liquids on nanostructurized surfaces behave in the opposite way to the described above. This results from the fact that there are air bubbles trapped in the cavities below the droplet which "sits" on the top of surface nanostructures. Such behaviour is described by the Cassie-Baxter model $[15,16]$. In our experiments the cavities were small and their cross-section in the shape of a reversed triangle, thus it would be much more diffi-

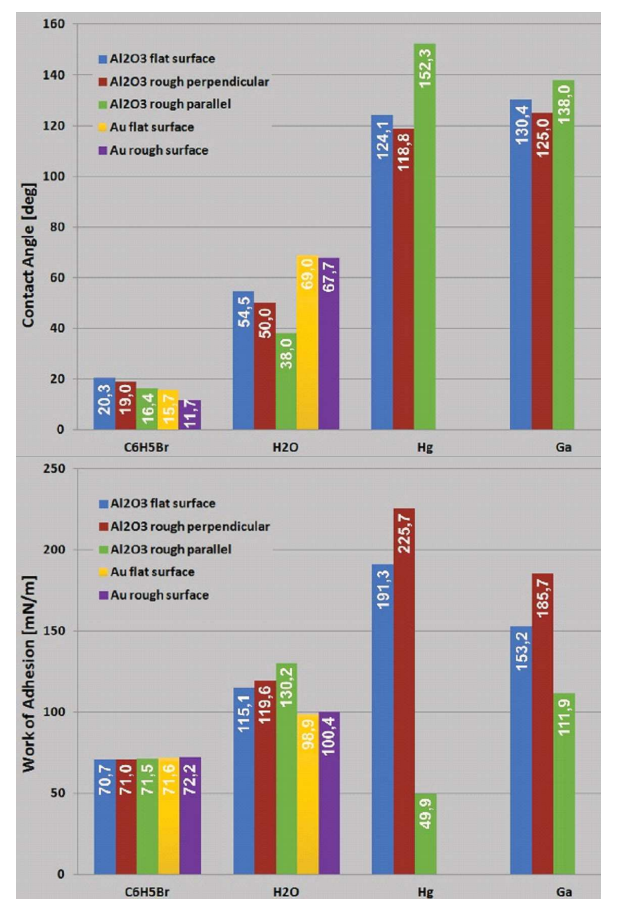

Fig. 4. Contact angle and work of adhesion values measured for liquid $\mathrm{C}_{6} \mathrm{H}_{5} \mathrm{Br}, \mathrm{H}_{2} \mathrm{O}, \mathrm{Hg}$ and $\mathrm{Ga}$ droplets placed on four surfaces: flat and nanostructurized sapphire and flat and nanostructurized gold (100 nm layer deposited on sapphire to retain the characteristic topography). Contact angle values of liquids on the nanostructurized sapphire differ when measured parallel and perpendicularly to the linear nanostructures. Results for mercury and gallium are presented only for sapphire surfaces, because the amalgam formation at the interface disrupts the measurements.

cult to trap gaseous phase below the droplet. This would explain why behaviour of our systems was more Wenzellike.

- There is a significant anisotropy of a contact angle on the nanostructurized sapphire. For $\mathrm{C}_{6} \mathrm{H}_{5} \mathrm{Br}$ and $\mathrm{H}_{2} \mathrm{O}$ the contact angle measured parallel to the nanogrooves is lower than measured perpendicularly (wetting is better along the stripes). In case of $\mathrm{Hg}$ and $\mathrm{Ga}$ on sapphire we observe the varying behaviour, depending on the direction of nanogrooves. The contact angle decreases when measured perpendicularly to the stripes and increases when measured parallel. Such behaviour does not fit the Wenzel rule of the amplification of wetting characteristics by roughening of the surface.

- There was no anisotropy of wetting in case of the goldcovered sapphire, despite that the topography remains very similar to that of the pure $\mathrm{Al}_{2} \mathrm{O}_{3}$. Two things differ the nanostructurized gold from the nanostructurized sapphire in our experiments: adhesion and roughness. Adhesion depends on the interaction between two materials in contact. Gold seems to be much different from sapphire in almost every way but at this stage of research we cannot confirm exactly which physical or chemical 
properties could be responsible for the observed differences in wetting of these surfaces. It might be easier to explain the observed lack of wetting anisotropy with the change of roughness as the gold surface used in this work has lower rms than sapphire. Due to the surface smoothing, the nanoscale difference between perpendicular and parallel direction might become insignificant for the "macroscale" contact angle measurements. To confirm this, further research on the surfaces with controlled roughness is needed.

\section{Conclusions}

In the presented work we focused on the influence of the nanometric surface roughness on the wetting phenomena. With four surfaces and four liquids we have shown several interesting aspects of wetting. The most obvious one is that wetting depended on the morphology of a surface, i.e. type of material and its nanoscale topography. Next, the directional roughening of the sapphire surface resulted in the anisotropy of the contact angle for all liquids used in this work. Contact angle measured in the direction perpendicular to the linear features on the solid surface decreases when compared to the value observed on the flat samples. In the parallel direction the effect depends on type of liquid. Lowsurface tension liquids show further decrease of the contact angle, while high-tension ones show the strong increase, even above the value observed on the flat surface. The most striking observation was that the contact angle on the directionally-roughened gold surface (thin layer of gold on the structurized sapphire) did not show any anisotropy at all. It might be due to the surface smoothening which occurred on the gold-covered sapphire, which would point at some critical level of roughness which is needed to make a liquid behave in the anisotropic way, but this effect needs further research to be fully explained.

Extensive research of the wetting phenomena are very important for many applications, where surfaces engineered in a specific way might significantly influence the wetting. Our results should prove useful in the research of porous materials, where the nanoscale roughness of the pore walls may be the key parameter for observed phenomena related to adsorption such as a shift of the phase transition temperatures and pressure enhancement effects.

\section{Acknowledgments}

We thank for financial support to National Science Centre, Grants No. DEC-2013/09/B/ST4/03711 (M.K. and M.Ś.-B.) and DEC-2013/11/B/ST3/04190 (J.J. and S.J.)

\section{References}

[1] J. Bico, C. Marzolin, D. Quéré, Europhys. Lett. 47, 220 (1999).

[2] E. Martines, K. Seunarine, M. Hywel, N. Gadegaard, C.D.W. Wilkinson, M.O. Riehle, Nano Lett. 5, 2097 (2005).

[3] F. Zhang, H.Y. Low, Langmuir 23, 7793 (2007).

[4] D. Quere, Annu. Rev. Mater. Res. 38, 71 (2008).

[5] R. Radhakrishnan, K.E. Gubbins, M. ŚliwińskaBartkowiak, J. Chem. Phys. 116, 1147 (2002).

[6] E.E. Santiso, A.M. George, M. Sliwinska-Bartkowiak, M.B. Nardelli, K.E. Gubbins, Adsorption 11, 349 (2005).

[7] Y. Long, M. Śliwińska-Bartkowiak, H. Drozdowski, M. Kempiński, K.A. Phillips, J.C. Palmer, K.E. Gubbins, Coll. Surf. A 437, 33 (2013).

[8] M. Śliwińska-Bartkowiak, A. Sterczyńska, Y. Long, K.E. Gubbins, Mol. Phys. 112, 2365 (2014).

[9] M.E. Schrader, Langmuir 11, 3585 (1995).

[10] R. Gabai, A. Ismach, E. Joselevich, Adv. Mater. 19, 1325 (2007).

[11] Surface tension values of some common test liquids for surface energy analysis.

[12] D.W. Ball, Physical Chemistry, Cengage Learning, 2014.

[13] B.J. Keene, Int. Mater. Rev. 138, 157 (1993).

[14] R.N. Wenzel, Ind. Eng. Chem. 28, 988 (1936).

[15] A.B.D. Cassie, S. Baxter, Trans. Faraday Soc. 40, 546 (1944).

[16] M.E. Abdelsalam, P.N. Bartlett, T. Kelf, J. Baumberg, Langmuir 21, 1753 (2005). 